\title{
Theoretical Study of General Base-catalyzed Hydrolysis of Aryl Esters and Implications for Enzymatic Reaction
}

\author{
Daiqian Xie, ${ }^{a}$ Dingguo Xu, ${ }^{b}$ Lidong Zhang, ${ }^{a}$ and Hua $G u{ }^{b}$ \\ ${ }^{a}$ Department of Chemistry, Institute of Theoretical and Computational Chemistry, Nanjing \\ University, Nanjing 210093, People's Republic of China \\ ${ }^{b}$ Department of Chemistry, University of New Mexico, Albuquerque, NM 87131, USA \\ (hguo@unm.edu)
}

Supporting information

Geometries of stationary points obtained at the B3LYP/6-31+G** level

I. RC for Model I

\begin{tabular}{|c|c|c|c|}
\hline C & 0.000000000000 & 0.000000000000 & 0.000000000000 \\
\hline $\mathrm{H}$ & 0.000000000000 & 0.000000000000 & 1.090338000000 \\
\hline $\mathrm{H}$ & 1.023940206144 & 0.000000000000 & $\cdot 0.386548562172$ \\
\hline C & .0 .729666460905 & 1.213035536851 & -0.504706686043 \\
\hline 0 & .0 .708176809378 & 1.248711558113 & $\cdot 1.880007210709$ \\
\hline 0 & $\cdot 1.281017530021$ & 2. 057229651029 & 0.165635240658 \\
\hline C & $\cdot 3.003735752101$ & 5.403061337861 & -4.982744881865 \\
\hline C & $\cdot 2.437776002399$ & 4.285702960916 & .4 .169893970082 \\
\hline C & $\cdot 2.732357364736$ & 2. 934752318487 & -4.408818923323 \\
\hline $\mathrm{H}$ & -3.399330015181 & 2.648423162753 & -5.215247679580 \\
\hline C & $\cdot 1.561975712271$ & 4.619913270722 & -3.123361953702 \\
\hline $\mathrm{H}$ & $\cdot 1.336133763025$ & 5.666098819261 & -2.948467464401 \\
\hline C & $\cdot 2.167146502036$ & 1.939660230631 & -3.615109195326 \\
\hline $\mathrm{H}$ & -2.381736979744 & 0.891211657871 & $\cdot 3.791782217363$ \\
\hline C & .0 .990773857435 & 3.632932572614 & $\cdot 2.325774767624$ \\
\hline $\mathrm{H}$ & .0 .320833600102 & 3.895021048450 & $\cdot 1.516248241266$ \\
\hline C & $\cdot 1.303872301136$ & 2. 295691973275 & $\cdot 2.578945112779$ \\
\hline $\mathrm{H}$ & .0 .489853257763 & 0.902753783557 & .0 .379073985825 \\
\hline 0 & .0 .848086157354 & 1. 101808574383 & 2.950350416109 \\
\hline $\mathrm{H}$ & $\cdot 1.037637649699$ & 1.565203993425 & 3.791066205404 \\
\hline $\mathrm{H}$ & $\cdot 1.156306950844$ & 1.680076878371 & 2.236293824050 \\
\hline $\mathrm{N}$ & $\cdot 1.586634235304$ & 2.366740724205 & 5.485470561120 \\
\hline$C$ & -1.030856434058 & 3. 314579729412 & 6.318543954781 \\
\hline C & .2 .673510677999 & 1.941145000065 & 6.097701168415 \\
\hline $\mathrm{N}$ & $\cdot 2.845328350785$ & 2. 575384962471 & 7. 293602327280 \\
\hline C & $\cdot 1.796502387386$ & 3.461356578614 & 7. 448900915027 \\
\hline $\mathrm{H}$ & $\cdot 0.118609096131$ & 3.829911053350 & 6.055941054544 \\
\hline $\mathrm{H}$ & $\cdot 3.355210026494$ & 1.189649374912 & 5.725881789046 \\
\hline $\mathrm{H}$ & $\cdot 1.696995605366$ & 4.090964619603 & 8.319224256422 \\
\hline $\mathrm{H}$ & -3.600346925797 & 2.423758793109 & 7. 945662721212 \\
\hline S & .4 .195759174381 & 4.940898499220 & -6.257895567149 \\
\hline 0 & -2.696543273637 & 6.569717175923 & -4.808722902927 \\
\hline
\end{tabular}


.4 .544140975433

$\mathrm{H} \quad-3.632956043278$

$\mathrm{H} \quad-5.274582014757$

II. TS for Model I

$\mathrm{H}$

$\mathrm{H}$

C

0

0

0

C

C

$\mathrm{H}$

C

$\mathrm{H}$

C

$\mathrm{H}$

C

H

C

S

C

$\mathrm{H}$

$\mathrm{H}$

$\mathrm{H}$

0

$\mathrm{H}$

H

$\mathrm{N}$

C

C

N

C

$\mathrm{H}$

$\mathrm{H}$

$\mathrm{H}$

$\mathrm{H}$
6.598765790214

7. 244373714192

7. 045373662241

6. 454652399171
.6 .931669379163

.6 .155880972072

.7 .331682209673

.7 .730234169402
0.00000000000
0.000000000000
0.000000000000
0.000000000000
0.000000000000
1. 094209000000
0.000000000000
1.028358053686
1. 216380986300
.0 .365854862508
.0 .775377921264
1. 188345486036
.0 .476211089902
.0 .463158519093
1. 440846992230
- 2.156286481226
- 1.944497985823
- 2.505845830038
.0 .164965198410
-2. 570238638775
-2. 204791489035
-3. 091543794932
.1 .335080074052
-2. 456216963199
$-0.853527820362$
- 1.158744173030
- 1.150368531971
- 3.072628109907
- 0.958558167882
-4. 074119360287
- 1.310039009963
- 0.510410254271
- 0.032469046619
0.308932887704
- 0.125633711773
0.172822605799
0.360114386175
$-6.894622415020$
.5 .830384420995
.4 .819438919742
. 5.079634696298
.6 .002690713662
- 3.611135297092
- 3.380205313846
.4 .170264542739
- 4.360913955507
.2 .696094354469
$-1.781632474623$
$-2.960022127973$
. 5.419831357704
- 2.842381821585
- 3.797546153648
.6 .940476120956
- 4. 250142151962
- 6.044956986680
- 3. 141839867585
- 5.052004193201
- 4.573981372154
- 4.302666310996
- 0.902118029846
0.137909678848
2. 454093585479
0.775432224010
2. 975313493291
3. 076299493385
3. 481128465234
3. 451837233123
4. 228763482554
.6 .818828773795
.7 .812303324657
.7 .065984198033
.0 .349025791881
.0 .236160199088
- 1.379100966472
0.112582783648
1. 325888185125
0.953037897438
2. 412920700384
4. 687177882742
- 2.230866528474
- 3.556084005363
2. 758750403714
4. 209132842389
- 2.108627448509
1. 849389140766
2. 878587818312
- 3. 328456201612
0.096639649965
4. 436808954539
-4. 257821734343
2. 940302815723
4. 440685368479
- 3.871997075055
1. 923200616250
5. 275296519337
- 1.190616455410
.5 .307926314406
3. 556262617883
- 3.525024832948

III. PC for Model I

$\begin{array}{ll}\mathrm{C} & 0.000000000000 \\ \mathrm{H} & 0.00000000000 \\ \mathrm{H} & 1.038124866452\end{array}$

0.000000000000

0.000000000000

0.000000000000

1. 092700000000

0.000000000000

- 0.348708642752 
$\cdot 0.670117951582$

2. 370751301581

- 1.388188346382

3. 451434889855

2. 638966396229

2. 538217327440

3. 421963690260

4. 139403764599

1. 608416269505

0.908395039941

3. 384907024817

4. 072256009105

1. 560991748334

0.833882983001

2. 451478264507

1. 472410389863

2. 006984697653

1. 363211967714

1. 888201589762

3. 050056323741

.0 .504296612531

.0 .372893546592

2. 937681008137

.0 .791201921783

3. 619452554930

4. 895790063411

2. 802213196325

3. 502318991711

4. 844959889804

5. 764801250348

1. 723777434232

5. 613882936384

3. 107628451284

IV. RC for Model II
1. 228730144427

1. 673592786583

1. 278217399498

5. 926674640294

5. 018769391232

4. 122542392877

4. 340882704696

5. 151225094623

3. 070918260371

2. 878442218216

3. 539634693531

3.718409295896

2. 264534200720

1. 464041237133

2. 489601733376

4.705251728580

6. 022518887006

5. 943754838970

7. 001716067193

5. 879987448136

$-0.886831362332$

2. 348293898949

1. 980668275072

3. 092958488721

2. 460214528236

2. 547839134985

2. 831577167922

3. 155140191329

2. 978257495940

2. 294809569679

2. 869166212382

3. 168335337987

3. 468861989353
.0 .545643603224

- 1.883207617092

- 1.519716243794

.6 .514616546949

- 6.427288614148

.5 .250302657460

.4 .178656337235

.4 .255283035726

- 5.149311849941

.5 .956308972848

- 3.044065224378

- 2. 223000914402

- 4.019898242381

.3 .932949543754

- 2.955714894313

$-7.779634027722$

.8 .920415424697

.9 .798970143132

. 8.454791582306

.9 .205902116423

$-0.383714193992$

0.181234015859

- 1.122513639746

- 0.284466898155

0.437308687295

0.954269457278

1. 406843826773

2. 528648747949

2. 255998756414

0.365129882422

1. 336454010525

2. 988146444969

3. 403077594479

0.000000000000

0.000000000000

0.000000000000

0.000000000000

1. 090195000000

1. 025778207794

0.000000000000

.0 .381902367773

1. 216303015931

.0 .507452396644

$-0.726157966672$

1. 241495815783

$-1.875446251613$

- 0.729544484548

2. 074095225842

0.171720187963

- 1.250034140295

5. 446747443536

.4 .886015874574

4. 339721166523

.2 .519695076997

3. 012866289830

.4 .082799042923

.4 .194631538357

2. 767425267912

.4 .884884081820

4. 617437245095

- 3. 187667706357

5. 638302552655

- 3.085350246696

1. 993011991551

- 3.434701472829 


$\begin{array}{ll}\mathrm{H} & -2.708352189510 \\ \mathrm{C} & -0.880753007624 \\ \mathrm{H} & -0.075742391612 \\ \mathrm{C} & -1.343773603090 \\ \mathrm{H} & -0.487605474832 \\ \mathrm{O} & -0.749353312378 \\ \mathrm{H} & -0.895613871609 \\ \mathrm{H} & -1.052682720163 \\ \mathrm{~N} & -1.353417010233 \\ \mathrm{C} & -0.762254799933 \\ \mathrm{C} & -2.416738333898 \\ \mathrm{~N} & -2.539353739508 \\ \mathrm{C} & -1.482175129881 \\ \mathrm{H} & 0.140826153377 \\ \mathrm{H} & -3.115066547385 \\ \mathrm{H} & -1.345980816929 \\ \mathrm{H} & -3.268059863456 \\ \mathrm{H} & -3.786934127265 \\ \mathrm{H} & -2.411606328750 \\ \mathrm{H} & -3.803426998856\end{array}$

V. TS for Model II

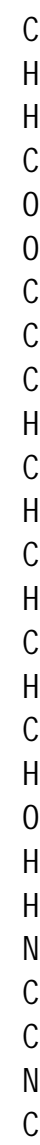

0.964684393797

3. 611977078275

3. 840082361833

2. 303538404689

- 0.902922263939

1. 233823824677

1. 784986615410

1.752768963548

2. 754643901962

3. 781331481225

2. 404875656894

3. 163802972808

4. 053280180678

4. 258761184036

1. 623347981604

4.770868654120

3. 087163185751

5. 045177219190

6. 102363631103

6. 074436065822
- 3.525737467334

-2. 424943525977

.1 .735604816236

- 2.554050639415

.0 .381094605050

2. 965848651493

3. 760098150674

2. 204458306420

5. 406361366183

6. 111589909604

6. 101908634745

7. 229679727701

7. 248959634260

5.760512116391

5.838699941879

8. 042889841711

7. 923411189519

.5 .690270170900

.5 .338321505258

- 4.253517634326
0.000000000000
0.000000000000
0.000000000000
1. 091710000000
0.000000000000
.0 .360408951000
1. 209778542942
2. 517056786136
1. 425003974905
4.590235558230
4. 032662463410
3. 421957823963
3. 346949483981
4. 101505944700
4. 567799161500
2. 894084069215
2. 404598735942
3. 596321113067
3. 652693881071
2. 978270596007
- 0.902452040926
1. 410397349879
2. 285744488241
1. 808926062729
2. 887980001264
3. 824520303046
2. 834771299250
3. 720499844768
4. 356064887862
- 0.571740536348
0.299809739359
- 1.774550349239
- 1.941378706376
- 1.350699429363
.0 .090587104889
0.481373957306
- 2.054937403520
- 3.038892567109
0.445018957213
1. 416143667804
- 1.528814075863
- 2.088257871947
.0 .258120052014
.0 .366344840239
0.214368491343
1. 389542918082
.0 .468780476933
2. 317760059643
2. 665654536622
3. 263064212895
4. 225464571813
3. 869087642467 
.0 .027070389213

.3 .613551587181

.0 .787041615333

-2. 957423912496

5. 806681950437

4.868777569861

5. 263735386661

VI. PC for Model II

$c$
$H$
0
0
$c$
$c$
$c$
$c$
$c$

3. 993191145358

2. 191344679847

5. 108620735719

3. 884245539321

4. 508730200617

5.649162256123

4. 056208560720
2. 017209026964

3. 268080574769

4. 490233400501

5.067622129289

.1 .235190003536

- 2.208763459576

.2 .855390237622
$\mathrm{C}$
$\mathrm{H}$
$\mathrm{H}$
$\mathrm{H}$
$\mathrm{C}$
$\mathrm{O}$
$\mathrm{O}$
$\mathrm{O}$
$\mathrm{C}$
$\mathrm{C}$
$\mathrm{C}$
$\mathrm{H}$
$\mathrm{H}$
$\mathrm{C}$
$\mathrm{H}$
$\mathrm{C}$
$\mathrm{C}$
$\mathrm{H}$
$\mathrm{C}$
$\mathrm{H}$
$\mathrm{H}$
$\mathrm{C}$
$\mathrm{H}$
$\mathrm{H}$
$\mathrm{O}$
$\mathrm{H}$
$\mathrm{H}$
$\mathrm{H}$
$\mathrm{N}$
$\mathrm{C}$
$\mathrm{C}$
$\mathrm{C}$
$\mathrm{N}$
$\mathrm{N}$
$\mathrm{C}$
$\mathrm{H}$
$\mathrm{H}$
$\mathrm{H}$
$\mathrm{H}$
$\mathrm{H}$
$\mathrm{H}$
$\mathrm{H}$
$\mathrm{H}$
$\mathrm{H}$
$\mathrm{H}$

VII. RC for Model III
0.000000000000
0.000000000000
1.097125000000
0.000000000000
0.000000000000
.0 .367662111868
1. 224690751022
$\cdot 0.504216316169$
- 0.211703042452
2. 173636184723
3. 888173216905
2. 802091757450
2. 279699929826
2. 663782585973
2. 276876855456
2. 657579167078
1. 278410636377
0.880552475627
1. 272572244434
0.885521216763
0.764547155641
- 0.904228642550
1. 186733348884
- 0.523812934468
2. 028980842933
- 1.152752579758
- 2. 031237144367
- 0.868279460357
- 1.528939014877
- 2.278306005951
- 2. 429597813113
- 0.210237784526
- 2.896906782617
- 1.479119820706
4. 789368431631
3. 561342048548
4. 177946834774
- 1.063253639589
7. 241432091900
6. 235279543155
5. 402540473352
5. 483535873653
6. 095181343244
6.723198243785
4. 472137655130
3. 836561588897
5. 165758481547
5. 075946039367
4. 344919673570
.0 .329487595593
- 0.254535430340
3. 414811563433
.0 .581994013189
3. 118902639470
3. 819016818632
2. 004953276679
1. 960343272802
3. 115924618121
4.777011388590
1. 220445377661
3. 322523324931
1. 213799934748
6.754963905735
7. 970181657665
7. 799174797865
C
0.000000000000
0.000000000000
0.000000000000
0.000000000000
1. 095988000000
H
1. 035806986558
0.000000000000
- 0.347386840081 
.0 .725784783962

0.106057246142

- 1.887982016662

- 2. 010929140663

- 1.449167655798

.0 .713949004361

- 0.524691106047

- 1.668745135515

- 2. 232928712671

- 0.217386444148

0.351421349250

- 1.178636566077

- 1.339541223015

- 0.460921123370

- 0.530825717923

$\cdot 0.737842584204$

0.130291545348

- 1.271597690777

1. 884744785729

2. 516449619385

2. 722164014062

3. 871808227804

3. 753280327647

2. 039243382619

2. 551127132838

4. 533479292339

4. 662580523818

- 1.556492000924

- 1.839426891144

.3 .094440000002

.4 .664310220723

-4. 459735474754

.4 .823913554752

- 3.831086373300
1. 240965443891

2. 331574860010

1. 305052768054

7. 591469422136

6. 199687260922

5. 574691570678

6. 105079770755

5. 488854012521

5. 955386283803

4. 276829057595

3. 792937647874

4. 195681212249

3. 641746896843

3. 602458469688

- 0.880135440091

1.691430521760

1.983864875401

1. 417615349398

2. 652307776442

3. 045122523224

2. 927794943468

3. 482558731412

3. 564061584129

2. 929413842078

2. 749574610552

3. 973071098925

3. 782982721294

8. 096312801702

8. 212111261723

7. 561781688693

2. 180128293223

3. 051909797791

1. 469513522741

1. 915587530478
.0 .437440870564

.0 .399658395944

.0 .766296417753

- 1.105402055230

.0 .922558333644

- 1.938065038817

$-2.868026319294$

0.268944202893

1. 073030678721

- 1.779283639890

- 2.567027928344

0.448085744613

1. 368370005041

.0 .588894788750

$-0.364354366499$

2. 820463938536

3. 180188961974

3. 575367821700

3. 406977273974

2. 243581829135

4. 387355254922

3. 907975620138

2. 533499580036

1. 281017441431

5. 439412792838

1. 910906190519

4. 458156807908

$-1.963171810224$

.0 .219127576000

- 1.275325992664

- 2.298903287719

.2 .779356243765

. 3.007665524938

- 1.777155557783

VIII. TS for Model III

$\begin{array}{lr}\mathrm{C} & 0.000000000000 \\ \mathrm{H} & 0.00000000000 \\ \mathrm{H} & 1.030805545448 \\ \mathrm{C} & -0.715435173959 \\ \mathrm{O} & 0.178241978927 \\ \mathrm{O} & -0.820647922428 \\ \mathrm{C} & 4.874464066913 \\ \mathrm{C} & 3.635199300658 \\ \mathrm{C} & 3.698670919198 \\ \mathrm{H} & 4.663684404418 \\ \mathrm{C} & 2.370256099022 \\ \mathrm{H} & 2.286799551198\end{array}$

0.000000000000

0.000000000000

0.000000000000

1.091632000000

0.000000000000

$-0.360297634122$

1. 208695715987

- 0.569685476762

2. 519158019519

0.297500462334

1. 427989821492

$-1.778295134233$

4. 654939282798

- 2.153417009010

4. 065061699489

- 1.517148852465

3. 350696255530

- 0.313418332122

3. 199313387419

0.167398484042

4. 229001223571

- 2. 106683311730

4.771242660622

- 3.046983102474 


$\begin{array}{lrrr}\mathrm{C} & 2.550402563467 & 2.817085257938 & 0.279044052073 \\ \mathrm{H} & 2.620212448827 & 2.248638723496 & 1.203474089315 \\ \mathrm{C} & 1.213614103693 & 3.716795105007 & -1.520208717952 \\ \mathrm{H} & 0.247303968888 & 3.847488366143 & -1.994285764858 \\ \mathrm{C} & 1.280395546576 & 2.996426891847 & -0.307209294872 \\ \mathrm{H} & -0.501397597937 & -0.903281343086 & -0.365097986356 \\ \mathrm{O} & -1.975595562840 & 1.427726616964 & 0.200045022262 \\ \mathrm{H} & -1.744750496509 & 2.358234057019 & 1.399722309026 \\ \mathrm{H} & -2.538727436959 & 1.833738315668 & -0.479940092572 \\ \mathrm{~N} & -1.740890250266 & 2.961523645831 & 2.304657049290 \\ \mathrm{C} & -0.762921817758 & 3.861433803606 & 2.663644007268 \\ \mathrm{C} & -2.693967717341 & 2.956381128404 & 3.226508818952 \\ \mathrm{~N} & -2.355944283087 & 3.839033963974 & 4.185442618466 \\ \mathrm{C} & -1.142439252609 & 4.421094668482 & 3.851634077401 \\ \mathrm{H} & 0.102232329607 & 3.988837356048 & 2.031942147491 \\ \mathrm{H} & -3.585524682564 & 2.349156095199 & 3.215825686018 \\ \mathrm{H} & -0.669670072772 & 5.161971884610 & 4.475349619157 \\ \mathrm{H} & -2.902728564780 & 4.036119138612 & 5.011752139912 \\ \mathrm{H} & 5.784499317161 & 4.274525189606 & -1.678186791063 \\ \mathrm{H} & 4.892445291023 & 5.749542908296 & -2.068406463569 \\ \mathrm{H} & 4.931784802025 & 4.415803614228 & -3.221822297720 \\ \mathrm{~N} & 0.867355955484 & 1.351327215867 & -4.433638511181 \\ \mathrm{H} & 1.817991455838 & 1.616396743940 & -4.189766988018 \\ \mathrm{H} & 0.893034961126 & 0.387401374898 & -4.755097377140 \\ \mathrm{H} & 0.319776304154 & 1.376077921944 & -3.572075251462\end{array}$

IX. PC for Model III

$\begin{array}{lrrr}\mathrm{C} & 0.000000000000 & 0.000000000000 & 0.000000000000 \\ \mathrm{H} & 0.000000000000 & 0.000000000000 & 1.096022000000 \\ \mathrm{H} & 1.029656503538 & 0.000000000000 & -0.358661256546 \\ \mathrm{C} & -0.706186036762 & 1.251760241461 & -0.462705021659 \\ \mathrm{O} & -1.590115311381 & 1.515952778743 & 3.336946873333 \\ \mathrm{O} & -0.132046271732 & 2.254855384777 & -0.853920132907 \\ \mathrm{C} & -3.963040122150 & 6.684010626286 & 2.829564049368 \\ \mathrm{C} & -3.350189227849 & 5.304718026153 & 2.932589590654 \\ \mathrm{C} & -1.959589426166 & 5.115651713539 & 2.888694631999 \\ \mathrm{H} & -1.309290386017 & 5.975640737514 & 2.742406394647 \\ \mathrm{C} & -4.146948717746 & 4.164981630052 & 3.118535401771 \\ \mathrm{H} & -5.229287807024 & 4.270649882324 & 3.163446402942 \\ \mathrm{C} & -1.384597754237 & 3.851942508731 & 3.025349743347 \\ \mathrm{H} & -0.308194938257 & 3.719915091160 & 2.978733967044 \\ \mathrm{C} & -3.589883240934 & 2.892064826492 & 3.259116497167 \\ \mathrm{H} & -4.230530216612 & 2.027340250049 & 3.401534590151 \\ \mathrm{C} & -2.195391429101 & 2.722237915180 & 3.212257104022 \\ \mathrm{H} & -0.523522856181 & -0.898448729274 & -0.337510941195 \\ \mathrm{O} & -2.043411375845 & 1.162937256277 & -0.361525784596 \\ \mathrm{H} & -2.245477474584 & 0.769312537571 & 3.315766660757 \\ \mathrm{H} & -2.441136962467 & 2.086662108735 & -0.516364759978\end{array}$




$\begin{array}{llll}\mathrm{N} & -3.320135297230 & -0.635641689400 & 2.992759806169 \\ \mathrm{C} & -3.949331942579 & -1.599048573224 & 3.753922105920 \\ \mathrm{C} & -3.585129273044 & -0.928867287546 & 1.731766670258 \\ \mathrm{~N} & -4.357575537399 & -2.047703660919 & 1.651535648177 \\ \mathrm{C} & -4.601848608582 & -2.488658235552 & 2.938394422119 \\ \mathrm{H} & -3.891686666844 & -1.594663938003 & 4.832578974059 \\ \mathrm{H} & -3.238919274609 & -0.369657622297 & 0.871747971699 \\ \mathrm{H} & -5.194608042182 & -3.365695983077 & 3.146019290692 \\ \mathrm{H} & -4.692982049652 & -2.474062932572 & 0.800463399719 \\ \mathrm{H} & -4.876075507807 & 6.678309690474 & 2.223249084329 \\ \mathrm{H} & -3.265895186834 & 7.396725987134 & 2.377188709446 \\ \mathrm{H} & -4.235506498650 & 7.078143482705 & 3.817385088128 \\ \mathrm{~N} & -2.876714033124 & 3.724035779860 & -0.620387789203 \\ \mathrm{H} & -2.951148553026 & 4.125403651465 & 0.313588313721 \\ \mathrm{H} & -3.671559015071 & 4.042453250613 & -1.167958467456 \\ \mathrm{H} & -2.023056594680 & 4.085983659213 & -1.042090429655\end{array}$

